\title{
Adaptação transcultural e validação da escala de Saúde Global do PROMIS para a língua portuguesa
}

\author{
Cross-cultural adaptation and validation of \\ the PROMIS Global Health scale in the \\ Portuguese language
}

\author{
Adaptación transcultural y validación de la \\ escala de Salud Global del PROMIS a la \\ lengua portuguesa
}

QUESTÕES METODOLÓGICAS

METHODOLOGICAL ISSUES

\author{
Camila Eugênia Zumpano 1 \\ Tânia Maria da Silva Mendonça 1 \\ Carlos Henrique Martins da Silva 1 \\ Helena Correia 2 \\ Benjamin Arnold 3 \\ Rogério de Melo Costa Pinto 1
}

\section{Resumo}

O objetivo deste estudo foi realizar a adaptação transcultural da escala de Saúde Global do Patient-Reported Outcomes Measurement Information System (PROMIS) para a lingua portuguesa. Os dez itens sobre Saúde Global foram adaptados transculturalmente por meio do método proposto pelo Functional Assessment of Chronic Illness Therapy (FACIT). A versão final do instrumento para a língua portuguesa foi autoadministrada em 1.010 participantes no Brasil. A precisão da escala foi verificada usando-se a análise dos efeitos piso e teto, confiabilidade da consistência interna e confiabilidade teste -reteste. Utilizou-se a análise fatorial exploratória e confirmatória para avaliação da validade de construto e dimensionalidade do instrumento. A calibração dos itens foi realizada por meio do Modelo de Resposta Gradual proposto por Samejima. Quatro itens globais necessitaram de ajustes após a realização do pré-teste. A análise das propriedades psicométricas demonstrou que a escala de Saúde Global tem boa confiabilidade, com coeficiente alfa de Cronbach de 0,83 e coeficiente de correlação intraclasse de 0,89. As análises fatorial exploratória e confirmatória revelaram um bom ajuste ao modelo previamente estabelecido de duas dimensões. As escalas de Saúde Física Global e Saúde Mental Global apresentaram uma boa cobertura do traço latente, de acordo com o Modelo de Resposta Gradual. Os itens Saúde Global do PROMIS para a língua portuguesa apresentaram equivalência em relação à versão original e propriedades psicométricas satisfatórias para a aplicação direcionada à população brasileira na prática clínica e em pesquisas.

Saúde Global; Qualidade de Vida; Psicometria; Estudos de Validação
Correspondência

C. E. Zumpano

Universidade Federal de Uberlândia.

Rua Alexandre Martins Marquez 290, Uberlândia, MG 38408-456, Brasil.

cazump@yahoo.com.br

1 Universidade Federal de Uberlândia, Uberlândia, Brasil.

2 Northwestern University, Evanston, U.S.A.

3 FACITtrans, Elmhurst, U.S.A. 


\section{Introdução}

Saúde é um "estado de completo bem-estar físico, mental e social e não apenas a ausência de doença ou enfermidade” 1 (p. 100). As medidas de saúde global são usadas para avaliar a perspectiva do próprio paciente acerca de diversos aspectos da sua saúde, ao contrário das avaliações específicas de funções, sintomas ou doenças 2 .

A aplicação das medidas de resultados relatados pelo paciente, que incorporam a perspectiva do próprio indivíduo acerca de sua saúde e tratamento, tem ganho relevância na prática clínica e em pesquisa, complementando as avaliações objetivas tradicionais 3,4,5.

Diante disso, o Patient-Reported Outcomes Measurement Information System (PROMIS), uma iniciativa do National Institutes of Health (NIH), dos Estados Unidos, revolucionou a área de medidas de resultados relatados pelo paciente ao fornecer bancos de itens precisos e válidos, de vários domínios da saúde, calibrados pela Teoria de Resposta ao Item (TRI) e destinados à avaliação do impacto de diversas doenças crônicas na qualidade de vida dos indivíduos 6,7.

Dentre os instrumentos do PROMIS, a escala de Saúde Global destaca-se por possibilitar coletar percepções gerais de saúde física, mental e social, com poucos itens e em tempo reduzido, em relação às medidas tradicionais 8,9 . Esse instrumento tem grande aplicabilidade, destina-se a uma variedade de doenças crônicas e é preditivo de eventos futuros importantes, tais como a utilização dos serviços de assistência à saúde e mortalidade 2 .

A escala de Saúde Global do PROMIS em sua versão original apresenta propriedades psicométricas adequadas, com valores de confiabilidade de 0,81 para a escala específica de Saúde Física Global e de 0,86 para a escala específica de Saúde Mental Global, que juntos requerem cerca de dois minutos para serem preenchidos pelo próprio indivíduo ${ }^{8}$.

Os itens originais do PROMIS foram desenvolvidos em inglês, o que inviabiliza o seu uso em países de outras línguas. Desse modo, a literatura recomenda o processo de tradução e adaptação transcultural desses itens, por meio de métodos rigorosos e amplamente utilizados, além da verificação da confiabilidade e validade para a sua utilização em outras culturas ou países 10,11,12,13,14. Após esses procedimentos, o instrumento pode ser utilizado em ensaios clínicos, estudos multicêntricos e na prática clínica de profissionais da saúde.

A escala de Saúde Global do PROMIS já foi traduzida para diversos idiomas como espanhol, holandês, francês, alemão, italiano e chinês 15. Assim, o objetivo deste estudo foi realizar a adaptação transcultural da escala de Saúde Global do PROMIS para a língua portuguesa.

\section{Métodos}

\section{Desenho do estudo}

Estudo transversal de adaptação transcultural da escala de Saúde Global do PROMIS para a língua portuguesa, realizado após autorização da PROMIS Health Organization (PHO).

\section{Instrumentos}

O short form Saúde Global do PROMIS é composto por 10 itens que avaliam aspectos de saúde física, mental e social, além de um item para a classificação da saúde geral, sendo administrado no formato papel e caneta ou por via eletrônica ${ }^{2}$. Esse instrumento é composto por duas escalas específicas, Saúde Física Global e Saúde Mental Global, cada uma composta por quatro itens. A escala de Saúde Física Global inclui itens de saúde física (Global 03), função física (Global 06), intensidade da dor (Global 07) e fadiga (Global 08). E a escala de Saúde Mental Global é constituída pelos itens de qualidade de vida (Global 02), saúde mental (Global 04), satisfação com atividades sociais e relacionamentos (Global 05) e problemas emocionais (Global 10). Dois itens, saúde geral (Global 01) e papéis sociais (Global 09), não são utilizados para a pontuação das escalas de Saúde Física Global e Saúde Mental Global. As categorias de respostas são escalas do tipo Likert de 5 pontos, exceto o item Global 07 que é avaliado por uma escala de 0 a 10, na qual o 0 significa ausência de dor e 10, presença da pior dor imaginável. 
Os itens globais 07, 08 e 10 tratam de conceitos negativos como, dor, fadiga e problemas emocionais, respectivamente, sendo necessária a recodificação dos seus escores para que altas pontuações reflitam melhor a saúde global. O escore desse instrumento varia de 10 a 50, sendo que quanto maior a pontuação, melhor a saúde global do respondente 8 .

Além desse instrumento, foi utilizado o World Health Organization Quality of Life Instruments-brief version (WHOQOL-BREF), que avalia a qualidade de vida e é validado para a população brasileira 16,17. É constituído por 26 questões, utiliza uma escala de resposta do tipo Likert de 5 pontos e foi usado a fim de verificar o grau de correlação de seus domínios físico e psicológico com as escalas de Saúde Física Global e Saúde Mental Global do PROMIS.

Também foi utilizado um questionário sociodemográfico e clínico concebido para o estudo a fim de caracterizar os participantes quanto às variáveis idade, gênero, grau de escolaridade, estado civil, raça e presença de doença crônica.

\section{Procedimentos}

A Figura 1 resume o processo de adaptação transcultural da escala de Saúde Global do PROMIS, que foi realizado em duas etapas principais: (1) tradução e avaliação da equivalência semântica, e (2) avaliação da equivalência de mensuração.

\section{Figura 1}

Fluxograma do processo de adaptação transcultural da escala de Saúde Global do PROMIS.

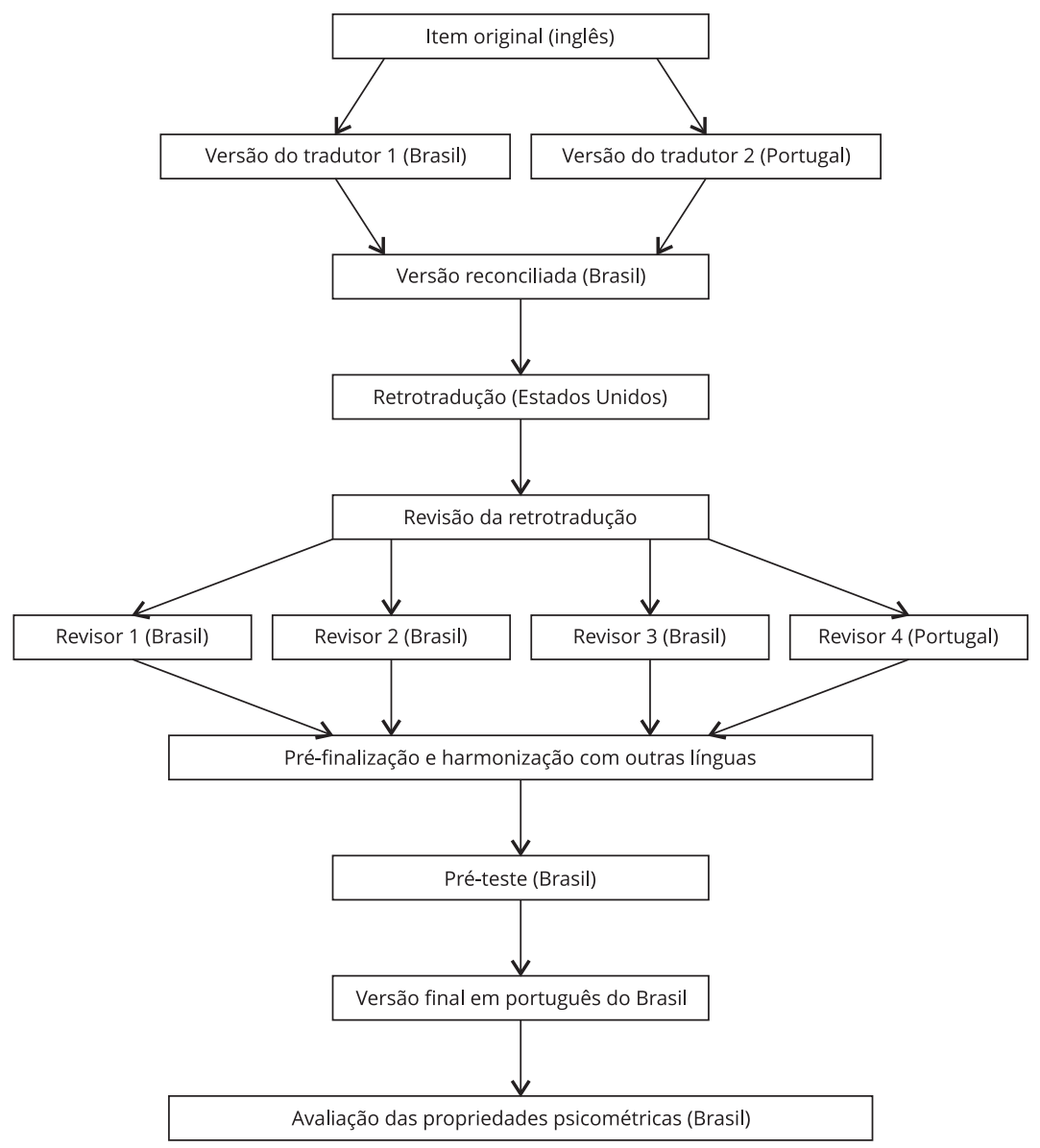

PROMIS: Patient-Reported Outcomes Measurement Information System. 


\section{- Tradução e avaliação da equivalência semântica}

Utilizou-se o método sistemático, de múltiplos estágios, e abordagem universal propostos pelo Functional Assessment of Chronic Illness Therapy (FACIT) e requerido pela PHO 13. A versão original em inglês dos itens sobre Saúde Global do PROMIS foi traduzida, simultaneamente, para a língua portuguesa por dois tradutores bilíngues independentes, fluentes nas línguas inglesa e portuguesa, um nativo do Brasil e o outro em Portugal. Em seguida, um terceiro tradutor bilíngue, brasileiro nativo e fluente em inglês, fez a reconciliação das duas traduções iniciais. A versão reconciliada foi retrotraduzida por um tradutor nativo usuário do inglês norte-americano, fluente na língua portuguesa, e sem conhecimento da versão original do instrumento. A versão retrotraduzida foi enviada à FACITtrans, empresa de tradução e validação linguística, licenciada pelo FACIT, e ao centro de coordenação de traduções do PROMIS na Universidade de Northwestern (Estados Unidos), para revisão e comentários. Logo após, quatro revisores bilíngues independentes, três nativos no Brasil e um nativo em Portugal, analisaram todas as etapas anteriores e selecionaram a tradução mais adequada para cada item ou forneceram traduções alternativas. O FACITtrans, o centro de coordenação de traduções do PROMIS e um coordenador de linguagem do Brasil foram responsáveis pela reconciliação entre as traduções propostas pelos revisores nativos do Brasil e em Portugal e a aprovação da versão para a fase de pré-teste.

\section{a) Participantes do pré-teste}

A versão pré-teste foi administrada em uma amostra de conveniência de 10 participantes 18 . Foram incluídos indivíduos com idades entre 18 e 60 anos, com doenças crônicas e em acompanhamento no Hospital de Clínicas da Universidade Federal de Uberlândia, localizado no Estado de Minas Gerais, Brasil. Esses participantes deveriam ter habilidades de escrita e leitura da língua portuguesa, verificadas pela aplicação de um item do instrumento selecionado de modo aleatório.

\section{b) Pré-teste}

O pré-teste foi realizado para identificar e corrigir possíveis problemas de tradução. Após o preenchimento do instrumento pelo próprio indivíduo, realizou-se uma entrevista retrospectiva de esclarecimento, para verificar se houve itens de difícil compreensão, irrelevantes ou ofensivos, e uma entrevista retrospectiva cognitiva, a fim de verificar a compreensão de cada item, por meio da técnica de sondagem verbal 19. O critério estabelecido para revisão e modificação da tradução foi compreensão dos itens por menos de $80 \%$ dos entrevistados ou sugestão de modificações por mais de $20 \%$ dos respondentes 20 .

\section{- Avaliação da equivalência de mensuração}

\section{a) Participantes}

A versão final em português do Brasil da escala de Saúde Global do PROMIS foi autoadministrada em uma amostra de conveniência de 1.010 participantes. Para garantir uma representação sociodemográfica e clínica heterogênea para calibração dos itens do instrumento, foram convidados a participar desta fase indivíduos com doenças crônicas ou saudáveis, com idades entre 18 e 60 anos, de ambos os sexos, distintas classificações étnico-raciais e níveis de escolaridade. Os indivíduos foram classificados como saudáveis ou com doença crônica por meio do autorrelato de doenças previamente existentes ou em acompanhamento. Os participantes deveriam ter habilidades de escrita e leitura da língua portuguesa, verificadas pela administração de um item do short form selecionado de modo aleatório, e assinar o termo de consentimento livre e esclarecido. Os indivíduos foram abordados, consecutivamente, no ambulatório de diversas especialidades médicas do Hospital de Clínicas da Universidade Federal de Uberlândia. O tamanho da amostra atende às recomendações para calibração dos itens pelo Modelo de Resposta Gradual proposto por Samejima 21, que exige cerca de 500 a 1.000 indivíduos 22,23. Além da versão final em português do Brasil da escala de Saúde Global do PROMIS, 
os participantes preencheram um questionário sociodemográfico e clínico e o WHOQOL-BREF, versão para a língua portuguesa 16,17 .

\section{Análise estatística}

\section{- Estatística descritiva}

Medidas de tendência central e dispersão foram utilizadas para a caracterização das variáveis sociodemográficas e clínicas dos pesquisados.

\section{- Qualidade dos dados}

Verificada por meio da análise dos dados perdidos e dos efeitos piso e teto. Os dados perdidos referem-se à proporção de respondentes que não completaram pelo menos um item da escala. Já os efeitos piso e teto representam a proporção de participantes que obtiveram os menores e maiores escores, respectivamente, em cada item da escala. Neste estudo, uma taxa de resposta igual ou superior a $80 \%$ foi avaliada como aceitável, e os efeitos piso e teto foram considerados presentes quando excederam a $10 \% 24,25$.

\section{- Confiabilidade}

Verificada usando-se o coeficiente alfa de Cronbach, sendo recomendado como padrão aceitável valores acima de 0,50 25. Para a análise da confiabilidade teste-reteste utilizou-se o coeficiente de correlação intraclasse (CCI), para o qual 100 participantes completaram a escala de Saúde Global após 14 dias da primeira aplicação. Em geral, valores de CCI acima de 0,75 são considerados adequados 26 .

\section{- Validade convergente}

Os itens sobre Saúde Global do PROMIS, bem como suas escalas específicas de Saúde Física Global e Saúde Mental Global, foram correlacionados com os domínios físico e psicológico do WHOQOL -BREF, versão para a língua portuguesa 16,17. O coeficiente de correlação de Spearman foi utilizado para a avaliação dessa validade. Um nível de correlação menor que 0,29 é considerado fraco, de 0,30 a 0,49 moderado e acima de 0,50 forte 27 .

\section{- Validade de construto e dimensionalidade}

A dimensionalidade consiste no número de fatores dominantes responsáveis por expressar o traço latente, ou seja, as características individuais não diretamente observáveis e avaliadas por meio das respostas aos itens do instrumento 28 . Neste estudo foram realizadas a análise fatorial exploratória (AFE) e análise fatorial confirmatória (AFC) para verificar a dimensionalidade do short form Saúde Global do PROMIS versão para a língua portuguesa, que é um pressuposto importante dos modelos da TRI. A AFE foi realizada para identificar a estrutura fatorial desta versão do instrumento. O índice Kaiser-Meyer-Olkin (KMO) e o teste de esfericidade de Bartlett foram usados para determinar a fatorabilidade da matriz de correlação. A variância total explicada pelo primeiro fator de pelo menos $20 \% \mathrm{e}$ autovalores acima de 1,0 foram os critérios utilizados para a determinação do número de dimensões 29 . O instrumento original é composto por duas escalas específicas, Saúde Física Global e Saúde Mental Global, cada uma composta por quatro itens, sendo que os dois itens restantes (Global 01 e Global 09) são analisados separadamente ${ }^{8}$. Realizou-se a AFC para verificar se o modelo de duas dimensões previamente estabelecido é ajustado aos dados 30 . O método de estimação Diagonally Weighted Least Squares (DWLS) e os índices de ajustamento dos modelos Comparative Fit Index (CFI), Tucker-Lewis Index (TLI), Root Mean Square Error Aproximation (RMSEA) e Standardized Root Mean Squared Residual (SRMR) foram utilizados para esta análise. Valores de CFI > 0,95; TLI > 0,95; RMSEA < 0,06 e SRMR $<0,08$ são recomendados como um bom ajuste do modelo 29,30,31,32. 


\section{- Calibração dos itens sobre Saúde Global do PROMIS}

A calibração dos itens sobre Saúde Global do PROMIS versão para a língua portuguesa foi realizada por meio do Modelo de Resposta Gradual proposto por Samejima 21 para cada escala, Saúde Física Global e Saúde Mental Global, separadamente, por se tratar de um instrumento multidimensional. Esse modelo da TRI permite estimar os parâmetros de dificuldade e discriminação do item, por meio do método de máxima verossimilhança marginal. Os parâmetros de dificuldade (b1, b2, b3, b4) correspondem ao valor do traço latente para o qual a probabilidade de responder a um determinado item é de $50 \%$, e posicionam os itens ao longo do continuum do construto. Já o parâmetro de discriminação (a) possibilita diferenciar os respondentes com níveis próximos do traço latente que está sendo aferido 29 . Valores do parâmetro de discriminação de 0,01 a 0,34 são considerados muito baixos; de 0,35 a 0,64 baixos; de 0,65 a 1,34 moderados; de 1,35 a 1,69 altos; e acima de 1,70 muito altos 33 .

Os softwares SPSS 17.0 (SPSS Inc., Chicago, Estados Unidos), LISREL 8.8 (Linear Structural Relationships. Jöreskog K, Sörbom D. Scientific Software International, Lincolnwood, Estados Unidos) e Multilog versão 7 (Scientific Software Inc., Chicago, Estados Unidos) foram utilizados para as análises estatísticas. O nível de significância estatística estabelecido foi de $\mathrm{p}<0,05$.

\section{Considerações éticas}

Este estudo foi aprovado pelo Comitê de Ética em Pesquisas com Seres Humanos da Universidade Federal de Uberlândia (CEP-UFU), sob o protocolo 443/10 e todos os participantes assinaram o Termo de Consentimento Livre e Esclarecido.

\section{Resultados}

\section{Tradução e avaliação da equivalência semântica}

Em relação ao processo de tradução, as discrepâncias mais frequentes entre os tradutores do Brasil e de Portugal foram quanto à inclusão do pronome pessoal de tratamento "você" em nove de dez itens, o uso do artigo definido antes do pronome possessivo, a inclusão da palavra "grau" nos itens globais 05, 07 e 08 e o uso da ênclise na tradução do item Global 10. A principal divergência entre os tradutores do Brasil e de Portugal está relacionada com a tradução de duas categorias de respostas, "regular" e "ruim", que não foram consideradas adequadas para Portugal. A equipe do FACITtrans, o centro de coordenação de traduções do PROMIS na Universidade de Northwestern e o coordenador de linguagem no Brasil foram responsáveis por resolver a maior parte das divergências, na tentativa de propiciar a harmonização entre a língua portuguesa do Brasil e de Portugal. Quaisquer diferenças irreconciliáveis restantes serão abordadas quando a versão de língua portuguesa da escala de Saúde Global for testada em Portugal.

Participaram do pré-teste 10 sujeitos com idade média de 35,6 anos $\pm 11,8,50 \%$ eram do sexo feminino, $50 \%$ brancos e $30 \%$ tinham menos que o ensino médio de escolaridade. Todos os pesquisados consideraram os itens relevantes, não ofensivos, de fácil entendimento e compreenderam as opções de respostas. Nenhum participante sugeriu modificações no instrumento. Os itens globais 05, 07, 08 e 09 necessitaram de ajustes após a realização do pré-teste. Esses itens foram novamente testados em uma amostra de cinco pacientes com doenças crônicas, a fim de verificar a compreensão dos mesmos após as modificações realizadas na tradução 18 . Esses quatro itens foram compreendidos por todos os participantes, garantindo equivalência conceitual e semântica com a versão original. Na Tabela 1 são apresentados todos os itens do instrumento em suas versões pré-teste e final, sendo explicadas as razões dos ajustes necessários nos quatro itens globais citados. A versão final da escala de Saúde Global do PROMIS para a língua portuguesa deve ser requisitada no Health Measures (http://www.healthmeasures.net) e na PHO. 
Tabela 1

Versões do pré-teste e final dos itens da escala de Saúde Global do PROMIS para a língua portuguesa após ajustes.

\begin{tabular}{|c|c|c|c|c|}
\hline Item & Versão original & Versão pré-teste & Versão final & Razão para ajuste \\
\hline Global 01 & In general, would you say your health is: & $\begin{array}{c}\text { De modo geral, você diria } \\
\text { que a sua saúde é: }\end{array}$ & $\begin{array}{l}\text { De modo geral, você diria } \\
\text { que a sua saúde é: }\end{array}$ & $\begin{array}{l}\text { Não houve necessidade de } \\
\text { ajuste após o pré-teste. }\end{array}$ \\
\hline Global 02 & $\begin{array}{l}\text { In general, would you say your quality } \\
\text { of life is: }\end{array}$ & $\begin{array}{c}\text { De modo geral, você diria } \\
\text { que a sua qualidade de } \\
\text { vida é: }\end{array}$ & $\begin{array}{l}\text { De modo geral, você diria } \\
\text { que a sua qualidade } \\
\text { de vida é: }\end{array}$ & $\begin{array}{l}\text { Não houve necessidade de } \\
\text { ajuste após o pré-teste. }\end{array}$ \\
\hline Global 03 & $\begin{array}{c}\text { In general, how would you rate your } \\
\text { physical health? }\end{array}$ & $\begin{array}{l}\text { De modo geral, como você } \\
\text { classificaria a sua } \\
\text { saúde física? }\end{array}$ & $\begin{array}{l}\text { De modo geral, como você } \\
\text { classificaria a sua } \\
\text { saúde física? }\end{array}$ & $\begin{array}{l}\text { Não houve necessidade de } \\
\text { ajuste após o pré-teste. }\end{array}$ \\
\hline Global 04 & $\begin{array}{l}\text { In general, how would you rate your mental } \\
\text { health, including your mood and your } \\
\text { ability to think? }\end{array}$ & $\begin{array}{l}\text { De modo geral, como você } \\
\text { classificaria a sua saúde } \\
\text { mental, incluindo seu } \\
\text { humor e sua capacidade } \\
\text { de raciocínio? }\end{array}$ & $\begin{array}{l}\text { De modo geral, como você } \\
\text { classificaria a sua saúde } \\
\text { mental, incluindo seu } \\
\text { humor e sua capacidade } \\
\text { de raciocínio? }\end{array}$ & $\begin{array}{l}\text { Não houve necessidade de } \\
\text { ajuste após o pré-teste. }\end{array}$ \\
\hline Global 05 & $\begin{array}{c}\text { In general, how would you rate your } \\
\text { satisfaction with your social activities and } \\
\text { relationships? }\end{array}$ & $\begin{array}{l}\text { De modo geral, como você } \\
\text { classificaria a sua satisfação } \\
\text { com atividades sociais } \\
\text { e relacionamentos? }\end{array}$ & $\begin{array}{c}\text { De modo geral, como você } \\
\text { classificaria a sua satisfação } \\
\text { com as atividades } \\
\text { sociais (atividades em } \\
\text { que se convive com } \\
\text { outras pessoas) e com } \\
\text { relacionamentos? }\end{array}$ & $\begin{array}{l}\text { Correspondência com } \\
\text { a tradução do Banco de } \\
\text { Itens Satisfação com a } \\
\text { Participação Social do } \\
\text { PROMIS versão para a } \\
\text { língua portuguesa. }\end{array}$ \\
\hline Global 06 & $\begin{array}{c}\text { To what extent are you able to carry out your } \\
\text { everyday physical activities such as walking, } \\
\text { climbing stairs, carrying groceries, } \\
\text { or moving a chair? }\end{array}$ & $\begin{array}{c}\text { Até que ponto você } \\
\text { consegue realizar } \\
\text { atividades físicas diárias } \\
\text { como andar, subir escadas, } \\
\text { carregar compras de } \\
\text { supermercado ou mover } \\
\text { uma cadeira? }\end{array}$ & $\begin{array}{c}\text { Até que ponto você } \\
\text { consegue realizar } \\
\text { atividades físicas diárias } \\
\text { como andar, subir escadas, } \\
\text { carregar compras de } \\
\text { supermercado ou mover } \\
\text { uma cadeira? }\end{array}$ & $\begin{array}{l}\text { Não houve necessidade de } \\
\text { ajuste após o pré-teste. }\end{array}$ \\
\hline Global 07 & How would you rate your pain on average? & $\begin{array}{l}\text { Em média, como você } \\
\text { classificaria a sua dor? }\end{array}$ & $\begin{array}{c}\text { Em média, como você } \\
\text { classificaria as suas dores? }\end{array}$ & $\begin{array}{l}\text { Compreensão do item pela } \\
\text { população brasileira de } \\
\text { modo distinto do conceito } \\
\text { original proposto. }\end{array}$ \\
\hline Global 08 & How would you rate your fatigue on average? & $\begin{array}{l}\text { Em média, como você } \\
\text { classificaria a sua fadiga? }\end{array}$ & $\begin{array}{c}\text { Em média, como você } \\
\text { classificaria o seu cansaço? }\end{array}$ & $\begin{array}{l}\text { Dificuldade de } \\
\text { compreensão da palavra } \\
\text { "fadiga" pela população } \\
\text { brasileira. }\end{array}$ \\
\hline Global 09 & $\begin{array}{l}\text { In general, please rate how well you carry out } \\
\text { your usual social activities and roles. (This } \\
\text { includes activities at home, at work and in } \\
\text { your community, and responsabilities as a } \\
\text { parent, child, spouse, employee, friend, etc) }\end{array}$ & $\begin{array}{l}\text { De modo geral, classifique } \\
\text { seu desempenho durante } \\
\text { a realização de atividades e } \\
\text { funções sociais frequentes } \\
\text { (incluindo atividades } \\
\text { em casa, no trabalho } \\
\text { e na comunidade, e } \\
\text { responsabilidades como } \\
\text { pai/mãe, filho/a, esposo/a, } \\
\text { empregado/a, amigo/a etc). }\end{array}$ & $\begin{array}{c}\text { De modo geral, classifique } \\
\text { a forma como consegue } \\
\text { desempenhar as suas } \\
\text { atividades e funções sociais } \\
\text { frequentes (incluindo } \\
\text { atividades em casa, no } \\
\text { trabalho e na comunidade, } \\
\text { e responsabilidades como } \\
\text { pai/mãe, filho/a, esposo/a, } \\
\text { empregado/a, amigo/a etc). }\end{array}$ & $\begin{array}{l}\text { Compreensão do item pela } \\
\text { população brasileira de } \\
\text { modo distinto do conceito } \\
\text { original proposto, }\end{array}$ \\
\hline Global 10 & $\begin{array}{c}\text { How often have you been bothered by } \\
\text { emotional problems such as feeling anxious, } \\
\text { depressed or irritable? }\end{array}$ & $\begin{array}{l}\text { Com que frequência você } \\
\text { se sentiu incomodado(a) } \\
\text { por problemas emocionais } \\
\text { como, por exemplo, sentir- } \\
\text { se ansioso(a), deprimido(a) }\end{array}$ & $\begin{array}{l}\text { Com que frequência você } \\
\text { se sentiu incomodado(a) } \\
\text { por problemas emocionais } \\
\text { como, por exemplo, sentir- } \\
\text { se ansioso(a), deprimido(a) }\end{array}$ & $\begin{array}{l}\text { Não houve necessidade de } \\
\text { ajuste após o pré-teste. }\end{array}$ \\
\hline & & & & \\
\hline
\end{tabular}

PROMIS: Patient-Reported Outcomes Measurement Information System. 


\section{Avaliação da equivalência de mensuração}

\section{- Caracterização dos participantes}

Participaram desta fase do estudo 1.010 sujeitos, sendo 511 indivíduos saudáveis (50,6\%) e 499 com doenças crônicas (49,4\%), com idade média de 36,3 anos, 50,1\% eram do sexo masculino, 43,5\% tinham entre 12 e 15 anos de escolaridade, 70,6\% eram casados e 69,5\% com classificação étnico-racial branca (Tabela 2). As doenças crônicas mais prevalentes autorrelatadas pelos participantes foram hipertensão (31,3\%), comprometimentos de coluna (19,9\%), diabetes $(11,3 \%)$ e enxaqueca $(8,8 \%)$.

\section{- Qualidade dos dados}

Não houve dados perdidos e efeito piso. Já o efeito teto foi observado em oito itens na amostra total da validação, em todos os itens no grupo de indivíduos saudáveis e em quatro itens no grupo de indivíduos doentes (Tabela 3).

\section{- Confiabilidade}

A escala de Saúde Global do PROMIS, versão para a língua portuguesa, e suas escalas específicas de Saúde Física Global e Saúde Mental Global apresentaram coeficientes alfa de Cronbach de 0,83, 0,63 e 0,65, respectivamente. O CCI foi de 0,89 ( $p=0,00$; IC95\%: 0,86-0,92).

\section{Tabela 2}

Características sociodemográficas dos participantes da avaliação da equivalência de mensuração $(n=1.010)$.

\begin{tabular}{lc}
\hline Características & Valor \\
\hline Idade média [anos] (DP) & $36,3(12,2)$ \\
Gênero - n (\%) & $506(50,1)$ \\
$\quad$ Masculino & \\
Escolaridade [anos] - n (\%) & $229(22,7)$ \\
$\quad$ Menos de 9 & $241(23,9)$ \\
$9-11$ & $439(43,5)$ \\
$12-15$ & $101(10,0)$ \\
Acima de 16 & \\
Estado civil - n (\%) & $714(70,6)$ \\
Casado & $56(5,5)$ \\
Separado & $223(22,1)$ \\
Solteiro & $17(1,7)$ \\
Viúvo & \\
Classificação étnico-racial - n (\%) & $2(0,2)$ \\
Amarela & $702(69,5)$ \\
Branca & $228(22,6)$ \\
Negra & $78(7,7)$ \\
Parda & \\
Número de doença crônica - n (\%) & \\
Nenhuma & $511(50,6)$ \\
1 & $386(38,2)$ \\
3 ou mais & $99(9,8)$ \\
\hline
\end{tabular}




\section{Tabela 3}

Efeito piso e efeito teto dos itens da escala de Saúde Global do PROMIS da amostra total da avaliação da equivalência de mensuração, do grupo de saudáveis e do grupo de doentes.

\begin{tabular}{|c|c|c|c|c|c|c|}
\hline \multirow[t]{2}{*}{ Item } & \multicolumn{2}{|c|}{ Amostra total $(n=1.010)$} & \multicolumn{2}{|c|}{ Grupo de saudáveis $(n=511)$} & \multicolumn{2}{|c|}{ Grupo de doentes $(n=499)$} \\
\hline & $\begin{array}{c}\text { Efeito piso } \\
n(\%)\end{array}$ & $\begin{array}{c}\text { Efeito teto } \\
\text { n (\%) }\end{array}$ & $\begin{array}{c}\text { Efeito piso } \\
n(\%)\end{array}$ & $\begin{array}{c}\text { Efeito teto } \\
\text { n (\%) }\end{array}$ & $\begin{array}{c}\text { Efeito piso } \\
\text { n (\%) }\end{array}$ & $\begin{array}{c}\text { Efeito teto } \\
\mathrm{n}(\%)\end{array}$ \\
\hline Global 01 & $31(3,1)$ & $166(16,4)$ & $9(1,8)$ & $135(26,4)$ & $22(4,4)$ & $31(6,2)$ \\
\hline Global 02 & $14(1,4)$ & $71(7,0)$ & $3(0,6)$ & $53(10,4)$ & $11(2,2)$ & $18(3,6)$ \\
\hline Global 03 & $33(3,3)$ & $75(7,4)$ & $9(1,8)$ & $55(10,8)$ & $24(4,8)$ & $20(4,0)$ \\
\hline Global 04 & $8(0,8)$ & $103(10,2)$ & $0(0,0)$ & $70(13,7)$ & $8(1,6)$ & $33(6,6)$ \\
\hline Global 05 & $9(0,9)$ & $126(12,5)$ & $1(0,2)$ & $97(19,0)$ & $8(1,6)$ & $29(5,8)$ \\
\hline Global 06 & $4(0,4)$ & $489(48,4)$ & $2(0,4)$ & $345(67,5)$ & $2(0,4)$ & $144(28,9)$ \\
\hline Global 07 & $46(4,6)$ & $216(21,4)$ & $7(1,4)$ & $154(30,1)$ & $39(7,8)$ & $62(12,4)$ \\
\hline Global 08 & $11(1,1)$ & $284(28,1)$ & $4(0,8)$ & $150(29,4)$ & $7(1,4)$ & $134(26,9)$ \\
\hline Global 09 & $1(0,1)$ & $141(14,0)$ & $0(0,0)$ & $106(20,7)$ & $1(0,2)$ & $35(7,0)$ \\
\hline Global 10 & $16(1,6)$ & $337(33,4)$ & $9(1,8)$ & $189(37,0)$ & $7(1,4)$ & $148(29,7)$ \\
\hline
\end{tabular}

PROMIS: Patient-Reported Outcomes Measurement Information System.

\section{- Validade convergente}

O teste de Kolmogorov-Smirnov indicou ausência de normalidade na distribuição da amostra ( $\mathrm{p}<0,01$ ), portanto, utilizou-se o coeficiente de correlação de Spearman para a avaliação desta validade. A correlação entre a escala de Saúde Global do PROMIS e os domínios físico e psicológico do WHOQOL-BREF foi significativa, de moderada a forte $(\mathrm{p}<0,01$; rho $=0,478$ e 0,571 , respectivamente). A escala de Saúde Física Global apresentou uma correlação moderada com o domínio físico do WHOQOL-BREF (rho = 0,461) e a escala de Saúde Mental Global apresentou uma correlação forte com o domínio psicológico deste instrumento ( $\mathrm{rho}=0,542$ ).

\section{- Validade de construto e dimensionalidade}

Os valores obtidos do índice de Kaiser-Meyer-Olkin (KMO =0,863) e do teste de esfericidade de Bartlett $\left(\chi^{2}=3.730,97 ; \mathrm{p}<0,000\right)$ revelaram uma correlação significativa entre os itens, confirmando a adequação da aplicação da AFE. A análise dos componentes principais com a presença de dois fatores, Saúde Física Global e Saúde Mental Global, explicou 56,96\% da variância total, sendo que 43,10\% foram explicadas pelo primeiro fator.

A AFC revelou um bom ajuste ao modelo previamente estabelecido de duas dimensões, já que produziu os seguintes índices de ajustamento, com valores muito próximos aos recomendados pela literatura 29,30,31,32: CFI = 0,95; TLI = 0,95; RMSEA = 0,13 (IC90\% = 0,12-0,14); e SRMR = 0,08. A correlação entre os dois fatores foi de $0,96(p<0,001)$.

\section{- Calibração dos itens sobre Saúde Global do PROMIS}

A Tabela 4 resume os parâmetros dos itens, discriminação e dificuldade, estimados para as escalas específicas de Saúde Física Global e Saúde Mental Global do PROMIS por meio do Modelo de Resposta Gradual proposto por Samejima 21. Na escala de Saúde Física Global, os valores do parâmetro de discriminação foram considerados de moderados a altos $(1,06$ a 1,44) e o item mais discriminativo foi o Global 07. Já na escala de Saúde Mental Global, os valores do parâmetro de discriminação foram de baixo a muito alto $(0,57$ a 3,06) e o item mais discriminativo foi o Global 04. O parâmetro de dificuldade do item variou de $-4,80$ a 2,30 na escala de Saúde Física Global e de -7,58 a 2,03 na escala de Saúde Mental Global. 
Tabela 4

Parâmetros dos itens das escalas de Saúde Física Global e Saúde Mental Global do PROMIS.

\begin{tabular}{|c|c|c|c|c|c|}
\hline Escala/Item & $\mathbf{a}$ & b1 & b2 & b3 & b4 \\
\hline \multicolumn{6}{|l|}{ Saúde física } \\
\hline Global 03 & 1,40 & $-3,06$ & $-1,09$ & 1,30 & 2,30 \\
\hline Global 06 & 1,42 & $-4,63$ & $-3,05$ & $-1,70$ & 0,07 \\
\hline Global 07 & 1,44 & $-2,70$ & $-1,04$ & 0,06 & 1,23 \\
\hline Global 08 & 1,06 & $-4,80$ & $-1,99$ & $-0,07$ & 1,10 \\
\hline \multicolumn{6}{|l|}{ Saúde mental } \\
\hline Global 02 & 1,94 & $-2,99$ & $-1,20$ & 1,08 & 2,03 \\
\hline Global 04 & 3,06 & $-2,79$ & 1,40 & 0,57 & 1,49 \\
\hline Global 05 & 1,91 & $-3,28$ & $-1,86$ & 0,12 & 1,55 \\
\hline Global 10 & 0,57 & $-7,58$ & $-3,43$ & 0,01 & 1,32 \\
\hline
\end{tabular}

Nota: $a$ = parâmetro de discriminação e b1, b2, b3 e b4 = parâmetros de dificuldade.

PROMIS: Patient-Reported Outcomes Measurement Information System.

\section{Discussão}

No presente estudo, foi descrito o processo de tradução e adaptação transcultural da escala de Saúde Global do PROMIS para a língua portuguesa, atendendo ao rigor metodológico preconizado na literatura internacional 12,13. Além disso, apresentou-se evidências que sustentam a adequação das propriedades psicométricas desta versão do instrumento para a população brasileira, conforme recomendado por seus desenvolvedores 29.

A versão final da escala de Saúde Global do PROMIS para a língua portuguesa, semelhante à de outros estudos de tradução 34,35,36,37, apresentou equivalência conceitual, semântica, cultural, operacional e de mensuração em relação aos itens originais no inglês.

Os resultados observados no presente estudo indicaram confiabilidade, validade e calibração dos itens pela TRI adequadas, assim como a versão original deste instrumento ${ }^{8}$. A escala de Saúde Global tem sido amplamente utilizada em diferentes contextos $38,39,40,41,42,43$, já que apresenta boas propriedades psicométricas e tempo reduzido para o preenchimento em relação às medidas tradicionais 44 .

Neste estudo, a ausência de dados perdidos e efeito piso indicou uma boa aceitabilidade desse instrumento para a população brasileira. A alta ocorrência do efeito teto no grupo de indivíduos saudáveis era esperada, uma vez que a maioria tem uma boa autopercepção do seu estado de saúde. A amostra de pessoas doentes pode explicar a ocorrência desse efeito nos itens de função física, dor, fadiga e problemas emocionais nesse grupo, visto que a coleta de dados foi realizada em ambulatórios de acompanhamento de doenças em estágios iniciais ou já bem controladas. O efeito teto também foi observado em todas as versões reduzidas do Banco de Itens Função Física do PROMIS em uma amostra diversa de pacientes com câncer 45. A administração do Banco de Itens Função Física do PROMIS em amostras de populações clínicas e gerais por meio do Teste Adaptativo Computadorizado reduz a ocorrência do efeito teto, uma vez que personaliza o instrumento para o nível de comprometimento do indivíduo, já que os itens serão selecionados de acordo com as respostas previamente dadas pelos sujeitos 46 . No entanto, esse modo de administração não está disponível para a escala de Saúde Global que é multidimensional e já por si é um short form.

A escala de Saúde Global do PROMIS, versão para a língua portuguesa, revelou ser precisa e reprodutível através do tempo, o que foi confirmado pelos valores adequados de coeficiente alfa de Cronbach e coeficiente de correlação intraclasse, respectivamente 25,26. Além disso, o instrumento demonstrou evidências de validade convergente, pois correlacionou-se em níveis de moderados a altos com os domínios físico e psicológico do WHOQOL-BREF, como era esperado, já que ambos, teoricamente, medem o mesmo construto. Os achados também sustentaram a estrutura de dois 
fatores do modelo previamente hipotetizado e semelhante à versão original do instrumento 8 , o que confirma a multidimensionalidade da escala de Saúde Global do PROMIS, que é representada por duas escalas específicas, Saúde Física Global e Saúde Mental Global.

As análises de confiabilidade e validade têm sido recomendadas pelos pesquisadores do PROMIS como forma de verificar se o instrumento é preciso e realmente mede o conceito alvo pretendido, e se sua estrutura fatorial está adequadamente representada, o que demonstra sua dimensionalidade, ou seja, o número de construtos dominantes que compõem o instrumento de avaliação 29,47.

A estimação dos parâmetros de dificuldade e discriminação dos itens, isto é, a calibração, é de fundamental importância, uma vez que possibilita posicionar os itens ao longo do continuum do traço latente e detectar diferenças entre os indivíduos avaliados. As escalas de Saúde Física Global e Saúde Mental Global apresentaram boa cobertura do traço latente subjacente. Além disso, ambas as escalas apresentaram itens com bons parâmetros de discriminação, o que possibilita diferenciar pessoas com distintos níveis do construto medido. Os itens menos discriminativos foram os que trataram de conceitos negativos, assim como na versão original do instrumento 8 .

Uma limitação deste estudo foi o número elevado de efeito teto detectado, o que poderia ser melhor avaliado em pesquisas com uma amostra mais específica, composta por pessoas com doenças crônicas com diversos níveis de comprometimento, uma vez que neste estudo os indivíduos apresentavam em sua maioria hipertensão, comprometimentos de coluna, diabetes e enxaqueca. Ressalta-se também a necessidade do processo de adaptação cultural da escala de Saúde Global do PROMIS em outros países que tenham a língua portuguesa como a oficial, diante de algumas divergências irreconciliáveis entre os tradutores nativos do Brasil e de Portugal, a exemplo do ocorrido na tradução de alguns instrumentos do PROMIS para o holandês e o flamengo 37 .

Os resultados encontrados demonstraram que esse instrumento é confiável e válido para aplicação no Brasil, e atende aos critérios de qualidade propostos para medidas de resultados relatados pelo paciente 48,49 .

\section{Conclusão}

A escala de Saúde Global do PROMIS versão para a língua portuguesa apresenta equivalência conceitual, semântica, cultural e operacional em relação aos itens originais, e propriedades psicométricas satisfatórias para a aplicação direcionada à população brasileira na prática clínica e em pesquisa.

\section{Colaboradores}

C. E. Zumpano, T. M. S. Mendonça, C. H. M. Silva e R. M. C. Pinto contribuíram na concepção do estudo, na análise e interpretação dos dados, na redação do artigo e aprovação final da versão a ser publicada. H. Correia e B. Arnold contribuíram na revisão do artigo e na aprovação final da versão a ser publicada.

\section{Agradecimentos}

Os autores agradecem ao apoio financeiro concedido pela Fundação de Amparo à Pesquisa do Estado de Minas Gerais - FAPEMIG (PPM00306-08). E também expressam o seu agradecimento pelas valiosas contribuições e esclarecimentos de David Cella, PhD da Universidade de Northwestern, durante a realização deste estudo. 


\section{Referências}

1. World Health Organization. Constitution. New York: World Health Organization; 1946.

2. PROMIS Cooperative Group. Unpublished manual for the Patient-Reported Outcomes Measurement Information System (PROMIS) Version 1.1. http://www.nihpromis.org (acessado em 02/ Jun/2012).

3. Acquadro C, Berzon R, Dubois D, Leidy NK, Marquis $\mathrm{P}$, Revicki D, et al. Incorporating the patient's perspective into drug development and communication: an ad hoc task force report of the patientreported outcoes (PRO) harmonization group meeting at the food and drug administration, february 16, 2001. Value Health 2003; 6:522-31.

4. Doward LC, McKenna SP. Defining patient-reported outcomes. Value Health 2004; 7 Suppl 1:S4-8.

5. Lohr KN, Zebrack BJ. Using patient-reported outcomes in clinical practice: challenges and opportunities. Qual Life Res 2009; 18:99-107.

6. Ader DN. Developing the patient-reported outcomes measurement information system (PROMIS). Med Care 2007; 45(5 Suppl 1):S1-2.

7. Cella D, Yount S, Rothrock N, Gershon R, Cook $\mathrm{K}$, Reeve B. et al. The patient-reported outcomes measurement information system (PROMIS): progress of an NIH Roadmap Cooperative Group during its first two years. Med Care 2007; 45(5 Suppl 1):S3-11.

8. Hays RD, Bjorner JB, Revicki DA, Spritzer KL, Cella D. Development of physical and mental health summary scores from the patient-reported outcomes measurement information system (PROMIS) global items. Qual Life Res 2009; 18:873-80.

9. Revicki DA, Kawata AK, Harnam N, Chen WH, Hays RD, Cella D. Predicting EuroQol (EQ-5D) scores from the patient-reported outcomes measurement information system (PROMIS) global items and domain item banks in a United States sample. Qual Life Res 2009; 18:783-91.

10. Guillemin F, Bombardier C, Beaton D. Crosscultural adaptation of health-related quality of life measures: literature review and proposed guidelines. J Clin Epidemiol 1993; 6:1417-32.

11. Gandek B, Ware Jr. JE. Methods for validating and norming translations of health status questionnaires: the IQOLA project approach. J Clin Epidemiol 1998; 5:953-9.

12. Beaton DE, Bombardier C, Guillemin F, Ferraz MB. Guidelines for the process of cross-cultural adaptation of self-report measures. Spine 2000; 25:3186-91.

13. Eremenco SL, Cella D, Arnold BJ. A comprehensive method for the translation and cross-cultural validation of health status. Eval Health Prof 2005; 28:212-32.

14. Reichenheim ME, Moraes CL. Operacionalização de adaptação transcultural de instrumentos de aferição usados em epidemiologia. Rev Saúde Pública 2007; 41:665-73.

15. PROMIS Cooperative Group. Available translations. http://www.healthmeasures.net/exploremeasurement-systems/promis/intro-to-promis/ available-translations (acessado em 06/Set/2016).
16. Fleck MPA, Leal OF, Louzada S, Xavier M, Chachamovich GV, Vieira G, et al. Desenvolvimento da versão em português do instrumento de avaliação de Qualidade de Vida da Organização Mundial da Saúde (WHOQOL-100). Rev Bras Psiquiatr 1999; 21:19-28.

17. Fleck MPA, Louzada S, Xavier M, Chachamovick GV, Vieira G, Santos L, et al. Aplicação da versão em português do instrumento abreviado de avaliação da qualidade de vida "WHOQOL-BREF". Rev Saúde Pública 2000; 34:178-83.

18. De Walt DA, Rothrock N, Yount S, Stone AA. Evaluation of item candidates: the PROMIS qualitative item review. Med Care 2007; 45(5 Suppl 1): S12-21.

19. Willis BG. Cognitive interviewing: a tool for improving questionnaire design. Thousand Oaks: Sage Publications; 2005.

20. Ruperto N, Ravelli A, Pistorio A, Malattia C, Cavuto S, Gado-West L, et al. Cross-cultural adaptation and psychometric evaluation of the Childhood Health Assessment Questionnaire (CHAQ) and the Child Health Questionnaire (CHQ) in 32 countries. Review of the general methodology. Clin Exp Rheumatol 2001; 19(4 Suppl 23):S1-9.

21. Samejima F. Estimation of latent ability using a response pattern of graded scores. Chicago: Psychometrika Society; 1969. (Series Psychometrika Monograph Supplement, 17).

22. Reise SP, Yu J. Parameter recovery in the graded response model using MULTILOG. J Educational Measurement 1990; 27:133-44.

23. Kim S, Cohen AS. A comparison of linking and concurrent calibration under the graded response model. Appl Psychol Meas 2002; 26:25-41.

24. Cramer JA. Principles of health-related quality of life: assessment in clinical trials. Epilepsia 2002; 43:1084-95

25. McHorney CA, Ware JE, Lu JFR, Sherbourne CD. The MOS 36 item short-form healthy survey (SF36): III. Test of data quality, scaling assumptions and reliability across diverse patient groups. Med Care 1994; 32:40-66.

26. Nunnally JC, Bernstein IH. Psychometric theory. 3rd Ed. New York: McGraw-Hill; 1994.

27. Cohen JW. Statistical power analysis for the behavioral sciences. 2nd Ed. Hillsdale: Lawrence Erlbaum Associates; 1988

28. Health outcomes methodology. Med Care 2000; 38(9 Suppl 2):II7-13.

29. Reeve BB, Hays RD, Bjorner JB, Cook KF, Crane PK, Teresi JA, et al. Psychometric evaluation and calibration of health-related quality of life item banks: plans for the Patient-Reported Outcomes Measurement Information System (PROMIS). Med Care 2007; 45(5 Suppl 1):S22-31.

30. Kahn JH. Factor analysis in counseling psychology research, training, and practice: principles, advances, and applications. Couns Psychol 2006; 34:684-718.

31. Hooper D, Coughlan J, Mullen M. Structural equation modelling: guidelines for determining model fit. Electronic Journal of Business Research Methods 2008 ; 6:53-60. 
32. Hair JF, Black WC, Babin BJ, Anderson RE, Tatham RL. Multivariate data analysis. $6^{\text {th }}$ Ed. New Jersey: Prentice Hall; 2006.

33. Baker FB. The basics of item response theory. 2nd Ed. Washington DC: Eric Clearinghouse on Assessment and Evaluation; 2001.

34. Nagl M, Gramm L, Heyduck K, Glattacker M, Farin E. Development and psychometric evaluation of a german version of the PROMIS item banks for satisfaction with participation. Eval Health Prof 2015; 38:160-80.

35. Liu Y, Hinds PS, Wang J, Correia H, Du S, Ding J, et al. Translation and linguistic validation of the pediatric patient-reported outcomes measurement information system measures into simplified chinese using cognitive interviewing methodology. Cancer Nursing 2013; 36:368-76.

36. Paz SH, Spritzer KL, Morales LS, Hays RD. Evaluation of the patient-reported outcomes information system (PROMIS) Spanish-languagem physical functioning items. Qual Life Res 2013; 22: 1819-30.

37. Terwee CL, Roorda LD, Vet HCW, Dekker J, Westhovens R, van Leeuwen J, et al. Dutch-Flemish translation of 17item banks from the patientreported outcomes measurement information system (PROMIS). Qual Life Res 2014; 23:1733-41.

38. Fenton BW, Palmieri P, Diantonio G, Vongruenigen V. Application of patient-reported outcomes measurement information system to chronic pelvic pain. J Minim Invasive Gynecol 2011; 8: 189-93.

39. Barile JP, Reeve BB, Smith A, Zack MM, Mitchell SA, Kobau R, et al. Monitoring population health for Healthy People 2020: evaluation of the NIH PROMIS ${ }^{\circledR}$ Global Health, CDC Healthy Days, and satisfaction with life instruments. Qual Life Res 2013; 22:1201-11.

40. Marx BL, Rubin LH, Milley R, Hammerschlag R, Ackerman DL. A prospective patient-centerd data collection program at an Acupuncture and Oriental Medicine teaching clinic. J Altern Complement Med 2013; 19:410-5.

41. Bingener J, Sloan JA, Seisler DK, McConico AL Sharan PE, Farely DR, et al. PROMIS for Laparoscopy. J Gastroint Surg 2015; 19:917-26.
42. Doll KM, Kalinowski AK, Snavely AC, Irwin DE, Bensen JT, Bae-Jump VL, et al. Obesity is associated with worse quality of life in women with gynecologic malignancies: An opportunity to improve patient-centered outcomes. Cancer 2015; 121:395-402.

43. Hinami K, Smith J, Deamant CD, DuBeshter K, Trick WE. When do patient-reported outcome measures inform readmission risk? J Hosp Med 2015; 10:294-300.

44. Bryan S, Davis J, Broesch J, Doyle-Waters MM, Lewis S, McGrail K. Choosing your partner for the PROM: a review of evidence on patient-reported outcome meausres for use in primary and community care. Healthc Policy 2014; 10:38-51.

45. Jensen RE, Potosky AL, Reeve BB, Hahn E, Cella D, Fries J. Validation of the PROMIS physical function measures in a diverse US population-based cohort of cancer patients. Qual Life Res 2015; 24:2333-44.

46. Rose M, Bjorner JB, Gandek B, Bruce B, Fries JF, Ware Jr. JE. The PROMIS physical function item bank was calibrated to a standardized metric and show to improve measurement efficiency. J Clin Epidemiol 2014; 67:516-26.

47. Hays R, Moinpour C. Validity-responsiveness. Appendix 12. PROMIS instrument development and validation scientific standards. Version 2.0 2013. http://www.nihpromis.com/Documents/ PROMISStandards_Vers2.0_Final.pdf?AspxAut oDetectCookieSupport=1 (acessado em 28/Nov/ 2016).

48. Terwee CB, Bot SD, Boer MV, van der Windt DA, Knol DL, Dekker J, et al. Quality criteria were proposed for measurement properties of health stats questionnaires. J Clin Epidemiol 2007; 60:34-42.

49. Reeve BB, Wyrwich KW, Wu AW, Velikova G, Terwee CB, Snyder CF, et al. ISOQOL recommends minimum standards for patient-reported outcome measures used in patient-centered outcomes and comparative effectiveness research. Qual Life Res 2013; 22:1889-905 


\section{Abstract}

This study aimed to perform the cross-cultural adaptation and validation of the Patient-Reported Outcomes Measurement Information System (PROMIS) Global Health scale in the Portuguese language. The ten Global Health items were crossculturally adapted by the method proposed in the Functional Assessment of Chronic Illness Therapy (FACIT). The instrument's final version in Portuguese was self-administered by 1,010 participants in Brazil. The scale's precision was verified by floor and ceiling effects analysis, reliability of internal consistency, and test-retest reliability. Exploratory and confirmatory factor analyses were used to assess the construct's validity and instrument's dimensionality. Calibration of the items used the Gradual Response Model proposed by Samejima. Four global items required adjustments after the pretest. Analysis of the psychometric properties showed that the Global Health scale has good reliability, with Cronbach's alpha of 0.83 and intra-class correlation of 0.89. Exploratory and confirmatory factor analyses showed good fit in the previously established two-dimensional model. The Global Physical Health and Global Mental Health scale showed good latent trait coverage according to the Gradual Response Model. The PROMIS Global Health items showed equivalence in Portuguese compared to the original version and satisfactory psychometric properties for application in clinical practice and research in the Brazilian population.

Global Health; Quality of Life; Psychometrics; Validation Studies

\section{Resumen}

El objetivo de este estudio fue realizar la adaptación transcultural de la escala de Salud Global del Patient-Reported Outcomes Measurement Information System (PROMIS) a la lengua portuguesa. Los diez items sobre Salud Global se adaptaron transculturalmente mediante el método propuesto por el Functional Assessment of Chronic Illness Therapy (FACIT). La versión final del instrumento en lengua portuguesa fue autoadministrada en 1.010 participantes en Brasil. La precisión de la escala fue verificada usándose el análisis de los efectos suelo y techo, confiabilidad de la consistencia interna y confiabilidad test-retest. Se utilizó el análisis factorial exploratorio y confirmatorio para la evaluación de la validez del constructo y dimensionalidad del instrumento. La calibración de los ítems se realizó mediante el Modelo de Respuesta Gradual, propuesto por Samejima. Cuatro items globales necesitaron ajustes tras la realización del pre-test. El análisis de las propiedades psicométricas demostró que la escala de Salud Global tiene una buena confiabilidad, con el coeficiente alfa Cronbach de 0,83 y el coeficiente de correlación intraclase de 0,89. Los análisis factoriales exploratorio y confirmatorio revelaron un buen ajuste al modelo previamente establecido de dos dimensiones. Las escalas de Salud Física Global y Salud Mental Global presentaron una buena cobertura del trazo latente, de acuerdo con el Modelo de Respuesta Gradual. Los ítems Salud Global del PROMIS adaptados a la lengua portuguesa presentaron equivalencia, respecto a la versión original y propiedades psicométricas satisfactorias para la aplicación dirigida a la población brasileña en la práctica clínica y en investigaciones.

Salud Global; Calidad de Vida; Psicometría; Estudios de Validación
Recebido em 21/Jun/2016

Versão final reapresentada em 08/Out/2016

Aprovado em 25/Out/2016 